\title{
Does interpolated interference affect only the short-term store in a free recall task?
}

\author{
SAM S. RAKOVER \\ University of Haifa, Haifa, Israel 31999
}

\begin{abstract}
Counting backward by threes following list presentation depresses only the terminal segment of the serial position curve in a free recall task. It was found that if the list is presented a second time, the entire curve is reduced uniformly, not merely the terminal segment. The significance of this result is discussed with respect to the duplexity and unitary theories of memory.
\end{abstract}

The purpose of this study is to demonstrate that an interpolated task in a short-term free recall experiment affects both short- and long-term storage (STS and LTS), as proposed by the unitary theory of memory, and not merely STS, as suggested by the duplexity model. The duplexity view assumes that the recency segment of the serial position curve represents retrieval from STS, while other segments are retrieved from LTS. According to this theory, items are displaced from the limited capacity STS by incoming items. Recall of terminal items, which are stored in STS, should be impaired by an interference task given before free recall of a list. Other variables, such as presentation rate and word frequency, which are known to influence LTS, should not affect retrieval of the terminal items. For example, lower presentation rates tend to elevate the initial and middle segments of the serial position curve. This suggests that an interpolated task would affect only STS, while other variables affect only LTS. The unitary model predicts, on the other hand, that the closer an item is to the end of the list, the less likely it is to be forgotten. An interpolated task, by preventing rehearsal and increasing the time between presentation and free recall, enhances the probability of forgetting for all list items. Thus, while the duplexity theory predicts that such interference reduces recall only in the final segment of the serial position curve, the unitary theory suggests that all segments should be affected.

The principal supporting evidence for the duplexity theory is the following. Subjects were required to execute various interference tasks, following presentation of a list of items. It was found that interference depressed only the terminal segment of the serial position curve. This result was corroborated by subsequent studies (see reviews in Glanzer, 1972, and Murdock, 1974). Nevertheless, a number of researchers (e.g., Gruneberg, 1970, 1972; Wickelgren, 1973) have suggested that the experimental evidence against the

This research was funded through the University of Haifa. The author is grateful to Sara David for helping to conduct the experiment and to Dr. David Navon for his helpful suggestions. Special thanks go to Prof. Murray Glanzer for his help in clarifying certain points. unitary theory is not persuasive, since the result may be attributed to a "floor effect."

Glanzer and Cunitz's (1966) experiment is a typical case in which the floor effect was not avoided. A list of 15 items was presented only once; each item was shown for $1 \mathrm{sec}$ followed by a 2 -sec interval. Those who favor the unitary theory propose that the interpolated task affects only retrieval of terminal items, since recall of the remaining items has already decreased to an asymptotic level, leaving no room for further decline. They explain the decline in performance regarding nonterminal items to an asymptotic level by the retroactive interference of each item on its predecessor.

In order to adequately test the contradictory implications of the duplexity and unitary models, the proposed "floor effect" must be eliminated. This was done in the present experiment by elevating the serial position curve by a constant amount. A uniform elevation is mandatory in order to prevent contamination of the results. If the increase is unequal, the interpolated task effects may reflect other variables than those under scrutiny. The requirement of constant elevation of the serial position curve excludes almost any operation known to enhance free recall. For example, lower rates of presentation (Glanzer \& Cunitz, 1966; Murdock, 1962; Raymond, 1969) have a differential effect in immediate free recall, improving retention for all but the terminal items. Brodie and Prytulak (1975) found that lower presentation rates resulted in more rehearsals for initial items than for later items in a list. Moreover, after an interpolated task, free recall of the terminal items was reduced to a greater extent with a lower list presentation rate.

In the present experiment the serial position curve was elevated by a constant amount by presenting the same list twice before testing for free recall. The duplexity theory would be confirmed if an interpolated task depressed only the terminal items.

\section{METHOD}

Materials and Equipment
Each stimulus consisted of a black-on-white slide of one
typewritten CVC trigram nonsense syllable presented by a 
Kodak Carousel slide projector. Of five lists, the first was a practice run of seven syllables. The other four were test lists, each comprising 15 different syllables. The syllables were taken from Underwood and Schulz (1960, Appendix A, Glaze's Syllables, Range 80-100). Forty psychology students examined the syllables in order to exclude those having sounds similar to words in Hebrew or Arabic. From these, 67 syllables were chosen and assigned to the various lists at random.

\section{Design and Procedure}

Four different groups were tested in a 2 by 2 design. The first factor was free recall of the test lists; immediately after presentation, or with a 30 -sec delay, counting backward by threes. The second factor was the number of times, once or twice, the same list was presented preceding free recall. Instructions were read prior to presentation of the practice list. Subjects were informed that the first list was a practice one and the other four were test lists. All items were presented for $1 \mathrm{sec}$, with an interstimulus interval of about 1 sec. Subjects in the immediate group were given the standard free recall task instructions. Subjects in the delay groups were instructed to covertly count backward by threes from a number which was displayed after presentation of each different list. After performing the interpolated task for $30 \mathrm{sec}$, they were instructed to write down the number which they had reached, and then to free recall the list. For the twice groups, the list was presented a second time, $1 \mathrm{sec}$ after the termination of the first list presentation, and only then were subjects instructed to free recall. The four test lists were presented in succession with a 1.5 -min free recall period for each list. Subjects were tested in groups of three or four. The order of list presentation was chosen randomly for each group of subjects.

\section{Subjects}

Sixty-four undergraduates volunteered to participate in the experiment, 16 subjects per group. Age distribution and sex ratio were similar among the four groups.

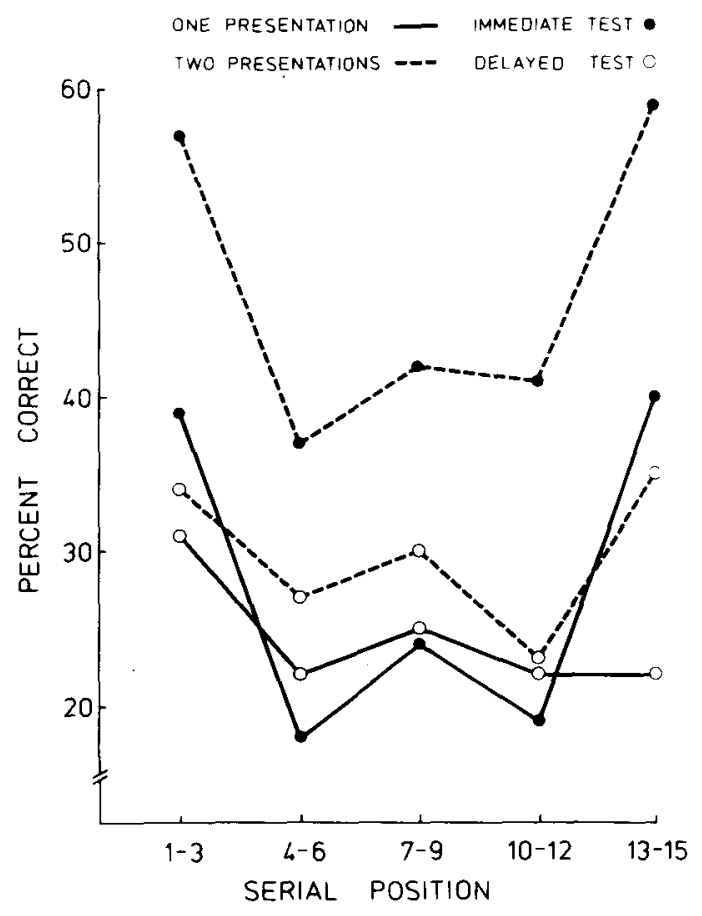

Figure 1. Mean percent of correct syllables recalled as a function of serial position and experimental condition.

\section{RESULTS}

Figure 1 depicts the mean percent of correct syllables recalled over the four test lists as a function of serial position (syllables grouped in threes) and the four experimental conditions. Utilizing a three-factor analysis of variance with serial position as a repeated factor, the following three main effects were found to be significant. First, the immediate groups recalled more correct syllables than the delay groups $[F(1,60)=15.65, p<$ $.01]$. Second, the twice groups recalled more correct syllables than the once groups $[F(1,60)=21.76, p<$ $.01]$. Third, on average a typical serial position curve was obtained $[F(4,240)=13.16, p<.01]$. Besides the three main effects, two interactions were found to be significant. Subjects recalled more correct syllables under the twice-immediate condition than under the other three conditions $[F(1,60)=6.65, p<.05]$. Furthermore, the serial position curve of the delay groups displayed a depressed recency section, in contrast to the immediate groups $[F(4,240)=3.95, p<.05]$.

As stipulated earlier, it was necessary to show that retention was enhanced by a uniform amount when a list was presented for a second time. An expected score was computed for each of the five serial positions in the once-immediate group in the following manner: A ratio of total number of syllables recalled in the once-immediate group over the total number of syllables recalled in the twice-immediate group was calculated. The ratio was then multiplied by scores obtained at each mean serial position of the twice-immediate group, giving five different products. Utilizing $t$ tests, no significant difference was found between any of the observed serial positions of the once-immediate group and its corresponding expected point.

The following analyses confirm that the interpolated task had a differential effect on the two delay groups. When comparing serial position scores of the onceimmediate group with corresponding points of the once-delay group, a t-test analysis demonstrated significant differences only at the terminal segment of the serial position curve $[t(30)=3.53, p<.01]$, the onceimmediate group recalling more than the once-delay group. This result constitutes a close replication of the results of Glanzer and Cunitz (1966) and Postman and Phillips (1965). However, the effect of the interpolated task on the twice groups was dissimilar. Each serial position point of the twice-delay group was reduced by a uniform amount from its corresponding point of the twice-immediate group. This conclusion was drawn by first computing the ratio of total number of syllables recalled in the twice-delay group over the total number of syllables recalled in the twice-immediate group. Then, expected serial position points for the twice-delay group were calculated by multiplying the computed ratio by the mean serial position points of the twice-immediate group. Utilizing $t$ tests, no significant differences were found between each observed serial position point of 
the twice-delay group and its corresponding expected point.

\section{DISCUSSION}

The results clearly support the unitary theory. The interpolated task depressed the terminal items of the list when it was presented only once, but reduced the total serial position curve when the list was presented twice. This finding is a direct prediction from the unitary model.

In order to attempt to interpret the present results by the duplexity model, one must first establish which part of the list was retrieved from which store. The assumption that the terminal items of the once groups are retrieved from either the STS alone or from both stores is compatible with the operational definition of short-term memory. The standard experimental paradigm is to present information only once, for a few seconds, and then test for retention after a few seconds. However, with the twice groups, since the lists were displayed a second time, the experimental procedure was not in accord with the operational definition of short-term memory. Therefore, the specific store from which the items were retrieved cannot be so easily established. Nevertheless, the present results can still be shown to be incompatible with duplexity model predictions. Whereas it is assumed that the initial portion of the serial position curve is retrieved from LTS and the latter portion is retrieved from STS when lists are presented only once, there exist nine possible combinations when lists are presented twice. The terminal portion may be retrieved from STS, LTS, or partially from STS and partially from LTS. Likewise, the same three possibilities can be postulated as sources of memory retrieval for the nonterminal portion of the serial position curve. If it is assumed that the present results are in accordance with the duplexity model supposition that an interference task affects only STS, then eight possible combinations can be readily discarded. Both the initial and terminal sections could not have been retrieved from LTS because the twice-delay condition resulted in a reduction of recall. In a like manner, the possibilities that the initial and terminal sections were retrieved from different storages, or that parts of each section were retrieved from different storages, can also be rejected because a constant depression was found in the twice-delay group, instead of an expected differential reduction. Thus, the present results may be compatible with the duplexity theory only if it is assumed that the whole list under the twice-delay was retrieved from STS.

Yet this interpretation is not convincing. First, the number of items recalled under the twice-delay condition is beyond STS capacity. Second, the number of items eliminated by the interpolated task in the twicedelay group is about five times more than the number of items ejected from the terminal section in the once- delay group. Third, the amount of information eliminated by the interpolated task in the twice-delay group is approximately twice STS capacity as estimated from the recency section of the once-immediate group. Finally, assuming that the discarded information in the twice-delay group did reside in STS, it is difficult to comprehend why the retention level of the terminal items of the twice-delay group was not lowered in a manner comparable to the once-delay group (see Figure 1).

In conclusion, the results of the present experiment cannot be explained by the duplexity theory. On the other hand, the results are in accordance with the unitary theory of memory, which assumes that retroactive interference reduces recall of the whole list. Furthermore, the findings and analyses substantiate the argument that counting backward affects only the recency section in the once-delay group because the initial items have already reached an asymptotic level of forgetting.

Nevertheless, the duplexity model should not be rejected entirely. Much evidence supports different aspects of the duplexity model. If the present experiment is taken as decisive, the future of the recency effect phenomenon may resemble that of the acousticsemantic dichotomy. It had been proposed that information is encoded phonetically in STS and encoded semantically in LTS. However, further research disclosed that encoding in each store can be by either modality depending on the demands of the specific task (e.g., Bjork, 1975; Wickelgren, 1973). Although encoding modality is no longer regarded as capable of differentiating between STS and LTS, much research is still based on the duplexity theory, which is viewed as a useful theoretical tool.

Given the present data, one may still consider the serial position curve as representative of two different stores. This experiment has merely demonstrated that an interpolated task affects the entire serial position curve. One could account for the results by postulating that interpolated tasks affect both memory stores, not just STS. However, other published memory research tends to contradict this supposition. Tzeng (1973), for example, suggests that a recency effect might not reflect retrieval from STS. He found this effect in both immediate and final recall of word lists when subjects counted backward by threes before and after each word presentation.

\section{REFERENCES}

BjoRk, R. A. Short-term storage: The ordered output of a central processor. In F. Restle, R. M. Shiffrin, N. J. Castellan, H. R. Lindman, \& D. B. Pisoni (Eds.), Cognitive theory (Vol. 1). Hillsdale, N. J: Lawrence Erlbaum, 1975.

Brodie. D. A.. \& Prytulak. L. S. Free recall curves: Nothing but rehearsing some items more or recalling them sooner? Journal of Verbal Learning and Verbal Behavior, 1975, 14, 549-563.

GlANZER, M. Storage mechanisms in recall. In G. H. Bower (Ed.), 
The psychology of learning and motivation: Advances in research and theory (Vol. 5). New York: Academic Press, 1972.

Glanzer, M., \& Cunitz, A. R. Two storage mechanisms in free recall. Journal of Verbal Learning and Verbal Behavior, 1966 , 5, 351-360.

GRUNEBERG, M. M. A dichotomous theory of memory-unproved and unprovable? Acta psychologica, 1970, 34, 489-496.

GRUNEBERG, M. M. The serial position curve and the distinction between short- and long-term memory. Acta psychologica, 1972, 36, 221-225.

Murdock, B. B., JR. The serial position effect of free recall. Journal of Experimental Psychology, 1962, 64, 482-488.

Murdock. B. B., JR. Human memory: Theory \& data. New York: Wiley, 1974.

Postman, L. , \& Phillips, L. W. Short-term temporal changes in free recall. Quarterly Journal of Experimental Psychology, 1965, $17,132-138$.
RAYmond, B. Short-term and long-term storage in free recall. Journal of Verbal Learning and Verbal Behavior, 1969, 8, 567.574

Tzeng, O. J. L. Positive recency effects in delayed free recall. Journal of Verbal Learning and Verbal Behavior, 1973, 12, 436-439.

UNDERWOOD, B. J., \& SchuLz, R. W. Meaningfulness and verbal learning. Philadelphia: J. B. Lippincott, 1960.

Wickelgren, W. A. The long and the short of memory. Psychological Bulletin, 1973, 80, 425-438.

(Received for publication March 30, 1977; accepted April 6, 1977.) 